\title{
Study on Ciprofibrate Equilibrium Solubility and Thermodymic Correlation in Four Aqueous Cosolvency Mixtures at Saturation
}

\author{
Guangyu Xu, Fanyuan Zhang, Zhenghui Li*
}

School of Materials and Chemical Engineering, Ningbo University of Technology, Ningbo, Zhejiang 315211,

People's Republic of China

Corresponding author. Phone: + 86574 87616023; Fax: + 8657487615880 .

E-mail address: zhenghui.li@nbut.edu.cn 


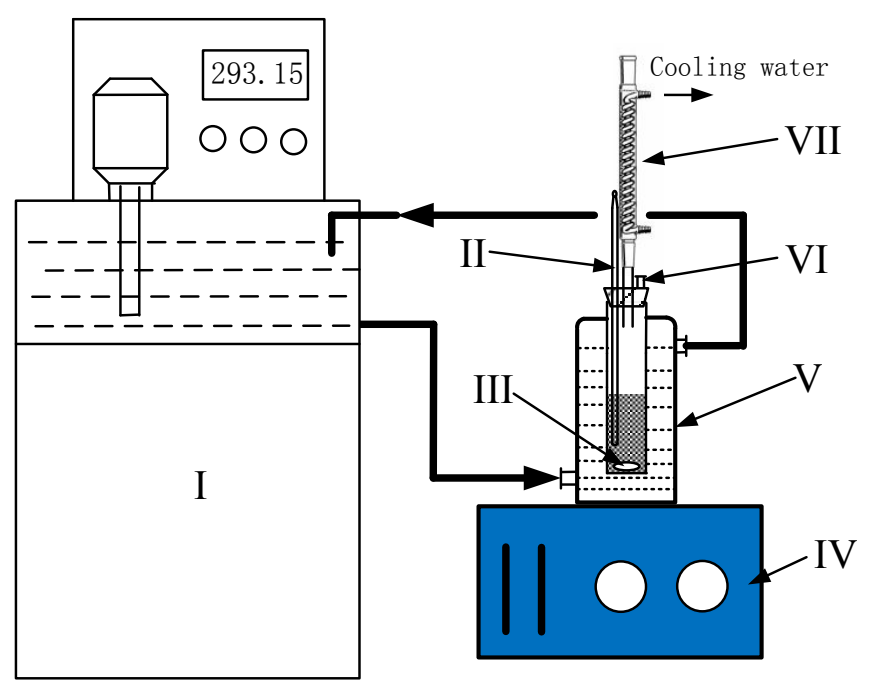

Fig. S1. Schematic diagram of experimental apparatus: I, smart thermostatic water bath; II, mercury-in-glass thermometer; III, magnetic stirrer; IV, stirrer controller; V, jacketed glass vessel; VI, sampling port; VII, condenser. 


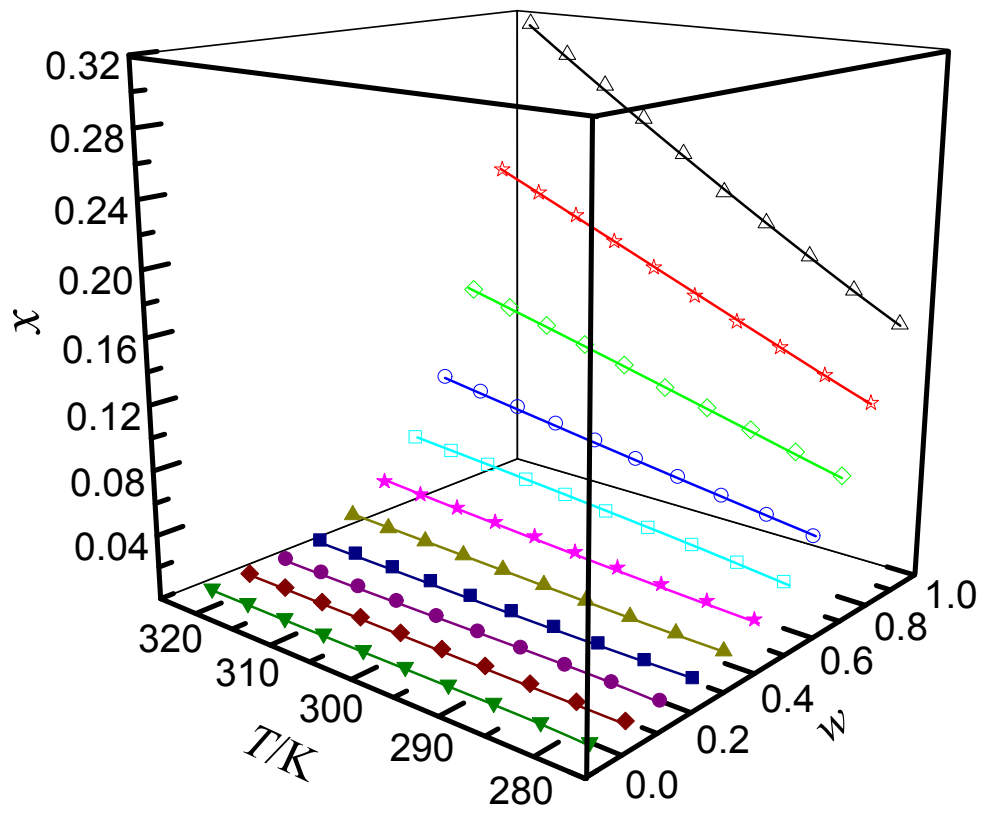

Fig S2. Experimental ciprofibrate solubility (in mole fraction) in ethanol $(w)+$ water $(1-w)$ cosolvent mixture with several mass fractions at temperature ranges of (278.15-323.15) $\mathrm{K}$ : $w$, mass fraction of ethanol; $\Delta, w=1.0 ;$ 访, $w=0.9 ; \diamond, w=0.8 ; \circ, w=0.7 ; \square, w=0.6 ; \star, w=0.5 ; \boldsymbol{\Delta}, w=0.4 ; \mathbf{\square}$, $w=0.3 ; \bullet, w=0.2 ; \bullet, w=0.1 ; \boldsymbol{\nabla}, w=0 ;-$, calculated plots by the Jouyban-Acree model. 


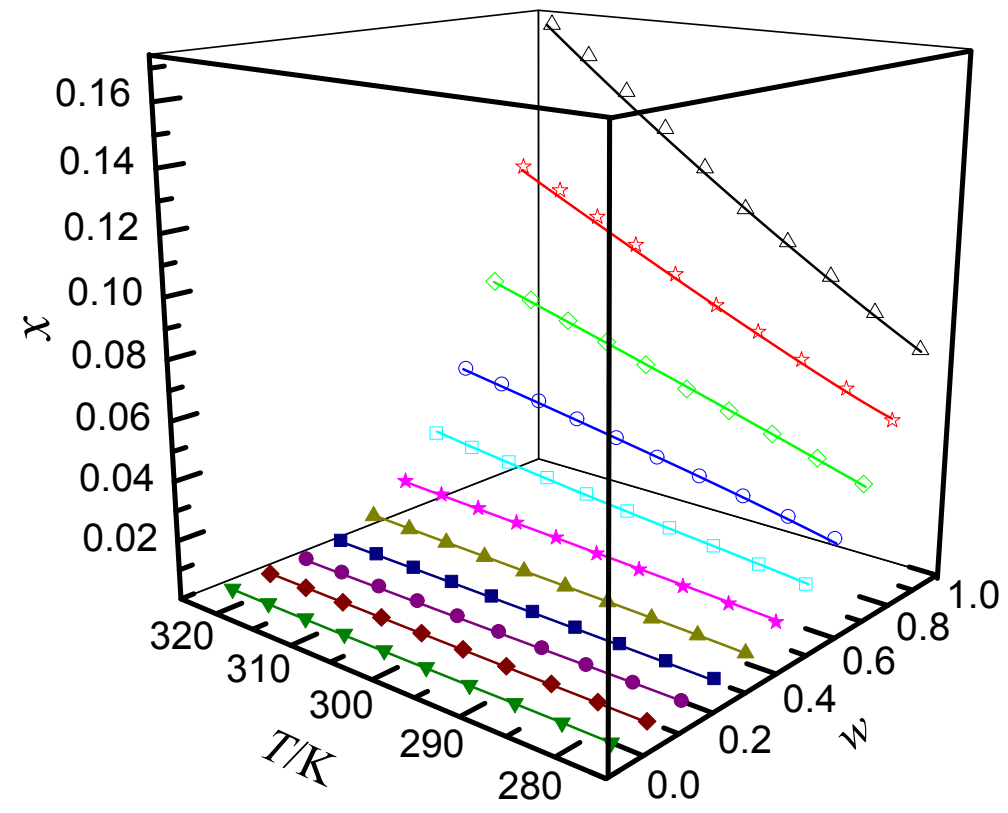

Fig S3. Experimental ciprofibrate solubility (in mole fraction) in $n$-propanol $(w)+$ water $(1-w)$ cosolvent mixture with several mass fractions at temperature ranges of (278.15-323.15) $\mathrm{K}$ : $w$, mass fraction of $n$-propanol; $\Delta, w=1.0 ; \downarrow, w=0.9 ; \diamond, w=0.8 ; \circ, w=0.7 ; \square, w=0.6 ; \star, w=0.5 ; \mathbf{\Delta}, w=0.4$; $\mathbf{\square}, w=0.3 ; \bullet, w=0.2 ; \bullet, w=0.1 ; \boldsymbol{\nabla}, w=0 ;-$, calculated plots by the Jouyban-Acree model. 


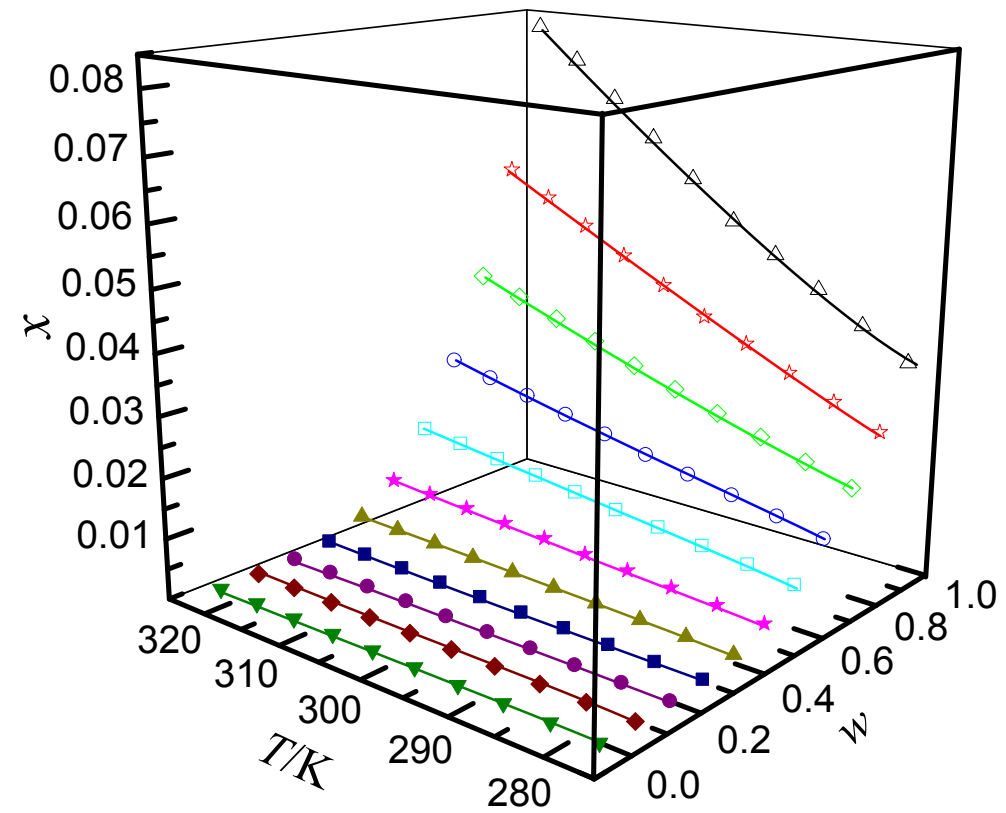

Fig S4. Experimental ciprofibrate solubility (in mole fraction) in EG $(w)+$ water $(1-w)$ cosolvent mixture with several mass fractions at temperature ranges of (278.15-323.15) $\mathrm{K}$ : $w$, mass fraction of EG; $\Delta, w=1.0 ;$ 计, $w=0.9 ; \diamond, w=0.8 ; \circ, w=0.7 ; \square, w=0.6 ; \star, w=0.5 ; \boldsymbol{\Delta}, w=0.4 ; \mathbf{\square}, w=0.3 ; \bullet$, $w=0.2 ; \diamond, w=0.1 ; \boldsymbol{\nabla}, w=0 ;-$, calculated plots by the Jouyban-Acree model. 


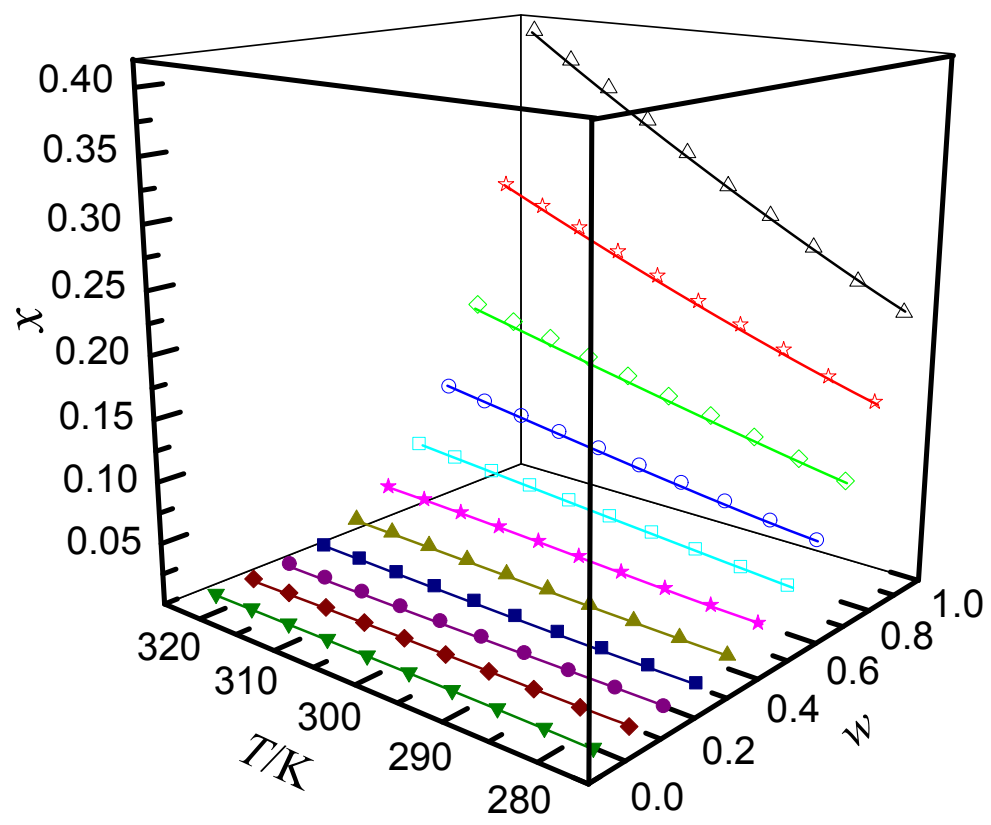

Fig S5. Experimental ciprofibrate solubility (in mole fraction) in DMF $(w)+$ water $(1-w)$ cosolvent mixture with several mass fractions at temperature ranges of (278.15-323.15) $\mathrm{K}$ : $w$, mass fraction of DMF; $\Delta, w=1.0 ;$ 访, $w=0.9 ; \diamond, w=0.8 ; \circ, w=0.7 ; \square, w=0.6 ; \star, w=0.5 ; \boldsymbol{\Delta}, w=0.4 ; \mathbf{\square}, w=0.3 ; \bullet$, $w=0.2 ; \diamond, w=0.1 ; \boldsymbol{\nabla}, w=0 ;-$, calculated plots by the Jouyban-Acree model. 